\title{
MULTIPACTING-FREE TRANSITIONS BETWEEN CAVITIES AND BEAM-PIPES*
}

SRF 080415-01

\author{
Sergey Belomestnykh and Valery Shemelin \\ Laboratory for Elementary-Particle Physics, Cornell University, Ithaca, NY 14853
}

\begin{abstract}
Recently multipacting (MP) has been experimentally found in two superconducting cavities to the surprise of experimenters. Computer simulation showed that the MP has occurred in transition regions between a cavity and a beam-pipe [2,3]. Our analysis offers an insight into which electromagnetic field configuration is necessary to support multipacting in such geometries. Namely, a minimum of the electric field along the cavity profile line, associated with the RF potential well, attracts electrons and thus creates conditions favorable for multipactor. Choosing geometries without a minimum of the electric field allows multipacting-free beam-pipe transitions. Simulation results confirming this conclusion are presented.
\end{abstract}

\section{INTRODUCTION}

Multipacting (MP) in an elliptic superconducting cavity can occur near the equator but usually it is not very strong and it was shown in [1] that one can shift the multipacting zone to higher fields by changing the cell shape in the equatorial region. It was thought that such cavities are essentially multipactor-free. However, recent tests of the Cornell ERL injector cavity [2] and KEK Ichiro cavity [3] showed relatively strong MP, which was later attributed, by performing computer simulations, to the transition regions between the cavity end cells and beam-pipes (Fig. 1). For the ERL cavity the presence of MP in this region was experimentally confirmed by a biased probe and correlated temperature changes at the outer wall [2]. Our analysis shows that the amplitude of the electric field along the cavity profile line has a minimum at the location of MP. Similarly, a possibility of MP existence in the transition region was found during our current work on designing a multicell superconducting cavity for the future Cornell ERL-based X-ray light source.

We proposed an explanation that an electric field minimum is associated with the local RF potential well, thus attracting electrons to its location and creating conditions favorable for MP. Simulations, performed using computer code MultiPac [4], confirm that smoothening out the transition to eliminate the minimum results in suppression of multipacting.

\section{CAVITY WITH TRANSITION FROM AN IRIS TO A LARGER DIAMETER BEAM- PIPE}

The transition from the cavity end cell to a beam-pipe is shown in Fig. 2. The contour line of this part of the

\footnotetext{
*Work is supported by NSF

\#vs65@ cornell.edu
}

multicell cavity consists of elliptic arcs connected with tangential straight segments. $A e, B e, A i, B i$ and so on are half-axes of the ellipses, $i$ refers to the inner half of the cell, $e$ refers to the outer half, $R$ is the radius of the circle smoothening the transition; $R e q$ is the equatorial radius, $R b p$ is the radius of the beam-pipe.
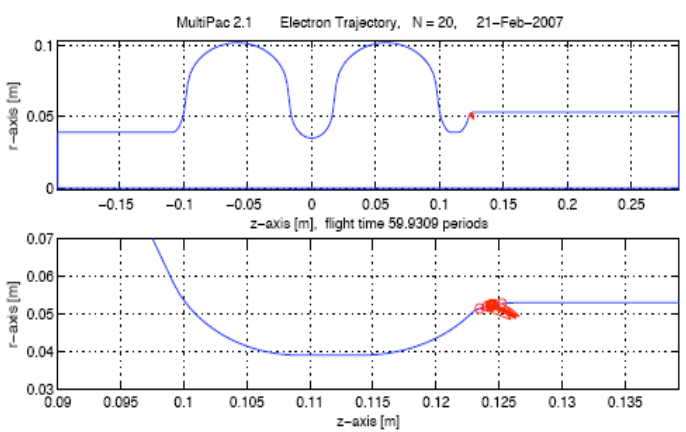

a)

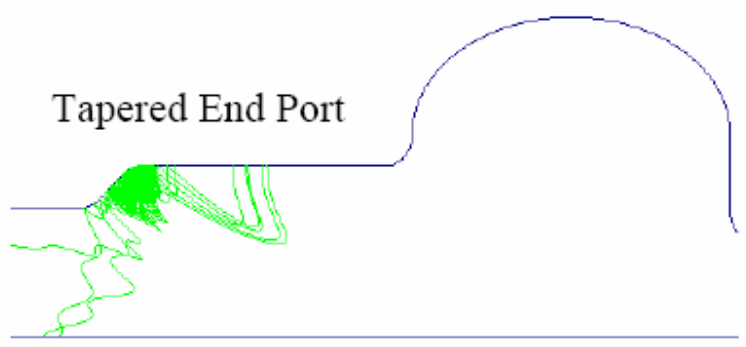

Figure 1: MP in the ERL Injector Cavity (a) and in the KEK Ichiro cavity (b).

We examined a transition from the end iris aperture $R a e=37 \mathrm{~mm}$ to the beam-pipe radius $R b p=55 \mathrm{~mm}$ with different radii $R$. Half-axes of the end iris ellipses were ae $=a t=12.53$ and $b t=b e=20.95 \mathrm{~mm}$. Other dimensions of the cavity are chosen to tune its frequency to $1300 \mathrm{MHz}$ and the ratio of the peak electric field to the accelerating field to $E_{p k} / E_{a c c}=2.0$.

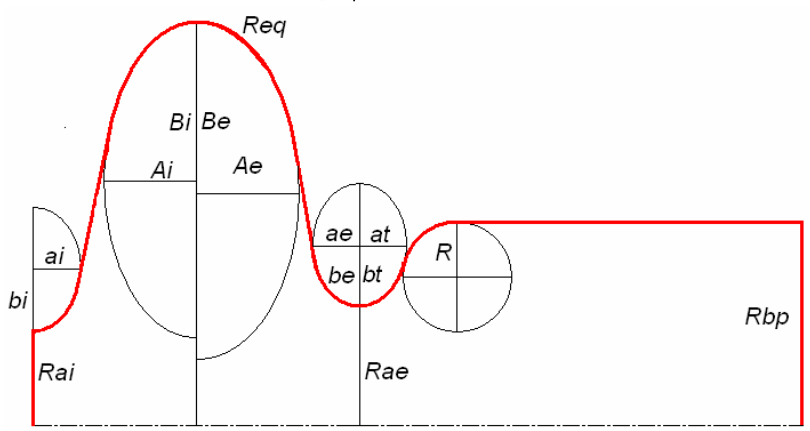

Figure 2: Geometry of the iris - beam-pipe transition.

Results of MP calculations for $R=9 \mathrm{~mm}$ are presented in Fig. 3. We can see stable trajectories with energy of 
primary electrons slightly too high for multipacting. The enhanced counter function [4] $e_{20} / c_{0}$, as designated on the graph, is about 0.1, i.e. less than unity that is necessary for a sustained discharge. (Actually, $e_{20} / c_{0}$ is a normalized enhanced counter function, as distinguished from the enhanced counter function $A$, which is the number of secondary electrons after a given number, $N=20$ in our case, of impacts. Normalization means that $A=e_{20}$ is divided by $c_{0}$, number of initial "seed" electrons distributed along the contour line with a given space and phase distribution relative to the field.) The data for the secondary electron yield was taken with a maximum equal to 1.5 , corresponding to a clean niobium surface. This is a typical situation when in an experiment the MP can initially occur, but is easily processed after
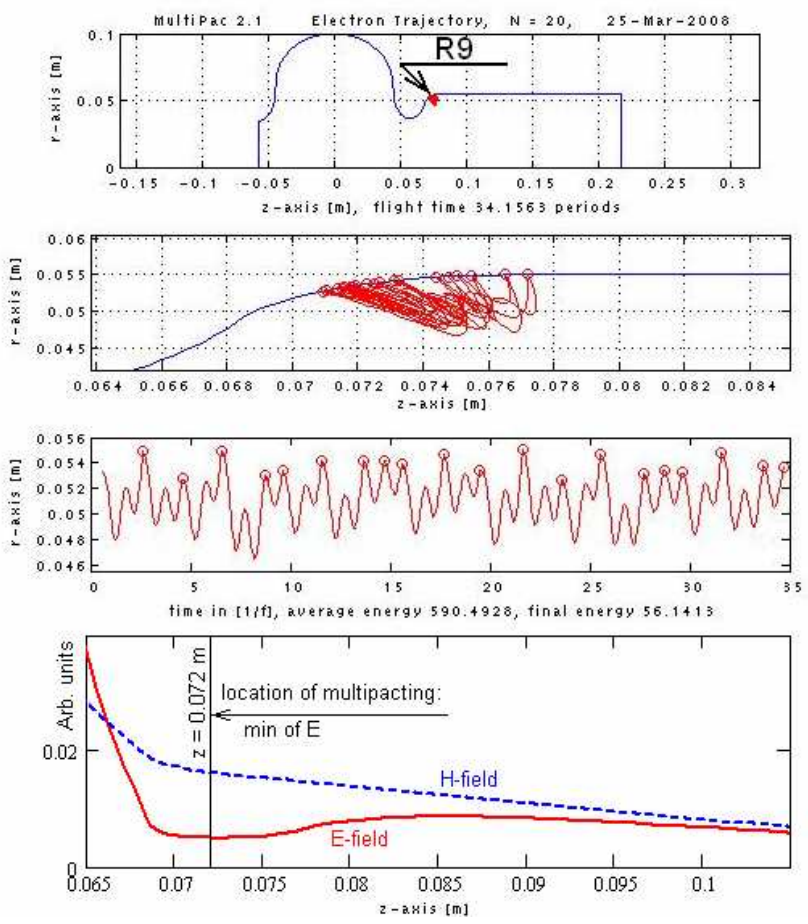

Figure 3: Multipacting simulation for a cavity having an inner corner rounded with $R=9 \mathrm{~mm}$.
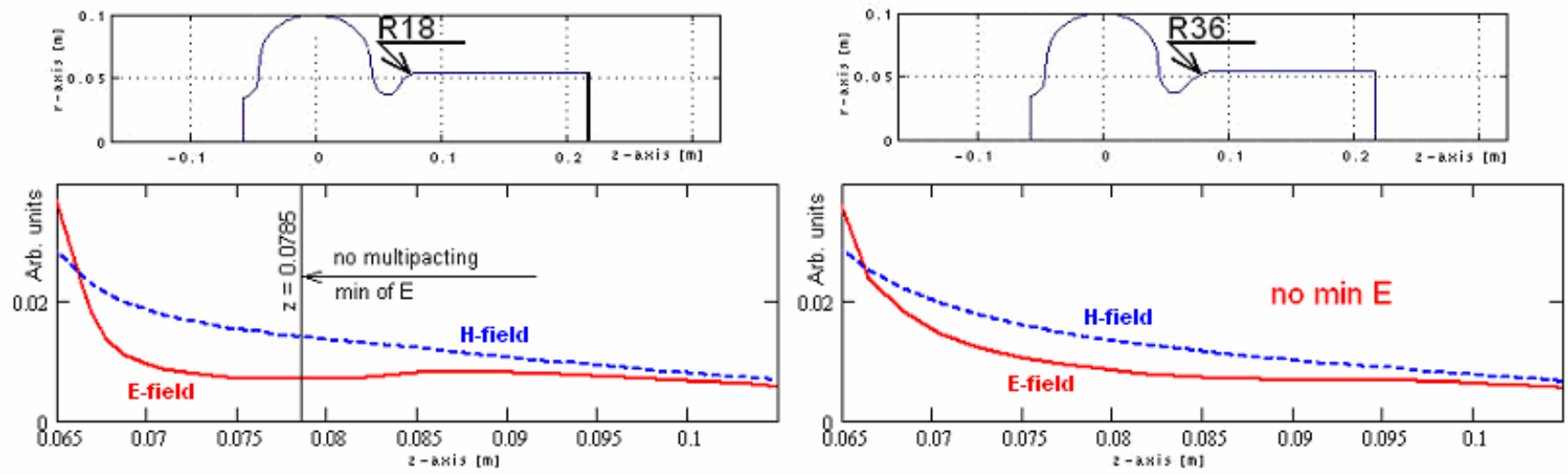

Figure 4: Minimum becomes shallow at $R=18 \mathrm{~mm}$ and disappears at $R=36 \mathrm{~mm}$. No MP in both cases. some operation with RF power when the surface becomes free of absorbates.

Let us emphasize that the location of the MP between $z=70$ and $76 \mathrm{~mm}$ coincides with the minimum of the electric field $E$ shown in the lower left plot in Fig. 3. The whole distribution of the fields along the profile line starting from the equator $(z=0)$ is shown in the lower right plot.

Increase of the radius $R$ to $18 \mathrm{~mm}$ eliminates MP though the very shallow minimum $E$ still exists, Fig. 4. This minimum disappears when the radius reaches $R=36$ $\mathrm{mm}$ (right-hand part of Fig. 4), which is 2 times the difference between $R b p$ and Rae (Fig. 2). This is possibly a sufficient condition to exclude MP in such transitions.

The change of the radius $R$ in the explored range changes the cavity resonant frequency by less than $1 \mathrm{kHz}$, the change of the $Q$ factor is negligible as well.
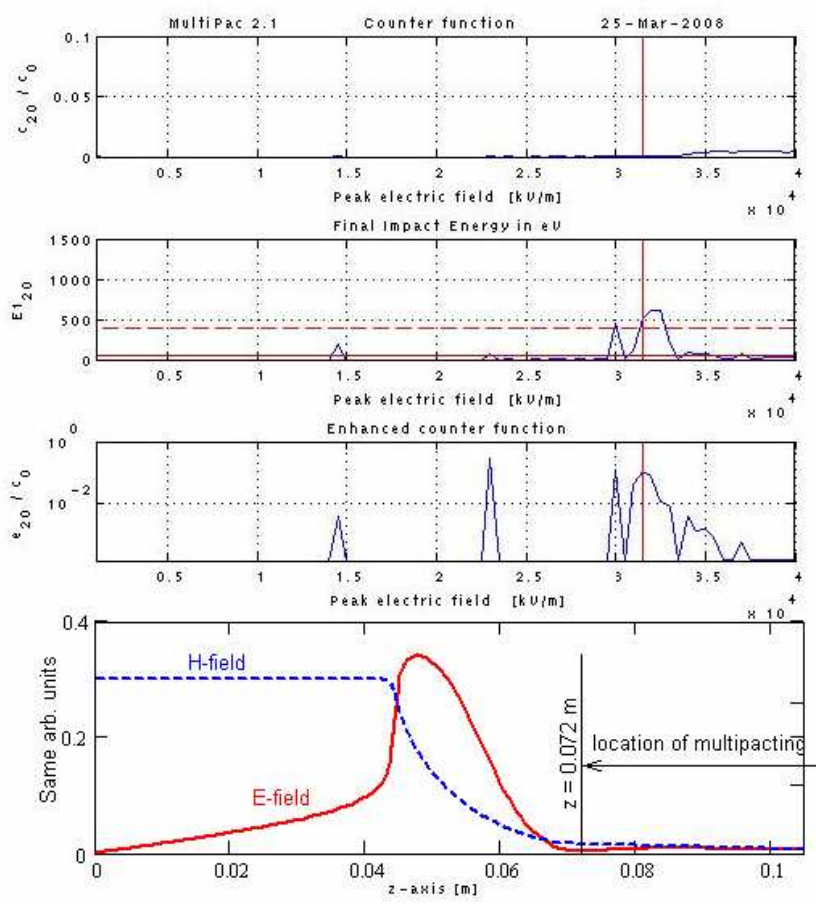
1 
A more detailed study indicates that there are several MP bands appearing at different field levels. Figs. 5 and 6 show dependence of the maxima of the enhanced counter function $A$ on the radius $R$ and corresponding values of the peak electric field $E$ as a function of $R$. Three sets of points in Fig. 5 and 6 correspond to three different bands of MP. Analyzed values of field levels were in the range from 25 to $35 \mathrm{MV} / \mathrm{m}$. This range of peak electric field was chosen for illustration because it has the most distinct maximum of the function $A$. Two points from Figures 5 and 6 corresponding to $R=12 \mathrm{~mm}$ are further analyzed in Fig. 7. There are presented two maxima of the normalized

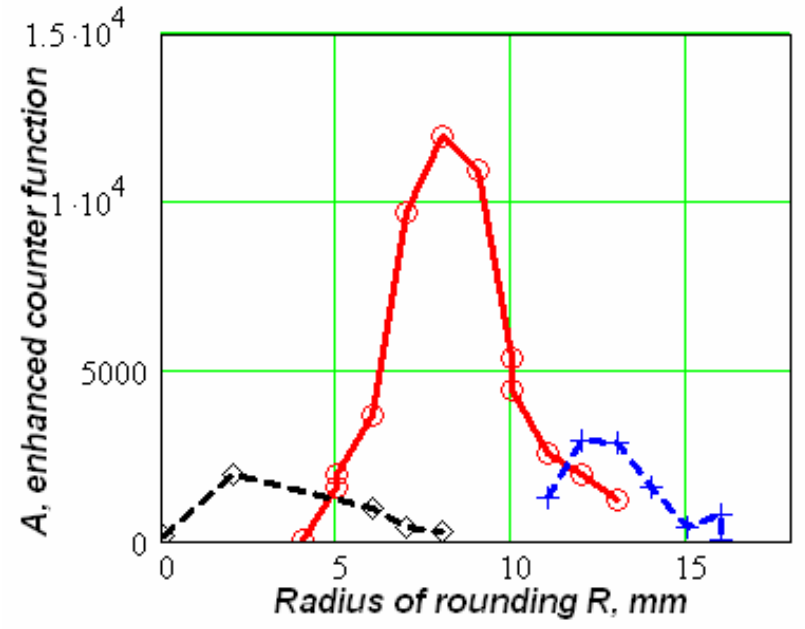

Figure. 5: Dependences of maximum $A$ on $R$. enhanced function $e_{20} / c_{0}$ and corresponding impact energies and trajectories. One can see that these trajectories can be related to two kinds of MP: threeperiodic for $25 \mathrm{MV} / \mathrm{m}$, and two-periodic MP for $33.5 \mathrm{MV} / \mathrm{m}$ (with some deviations from exact periodicity). Both kinds of MP are located in the flat minimum of the electric field. Our results are consistent with MP simulations for the Cornell ERL injector cavities with similar transition from the iris to large beam-pipe.

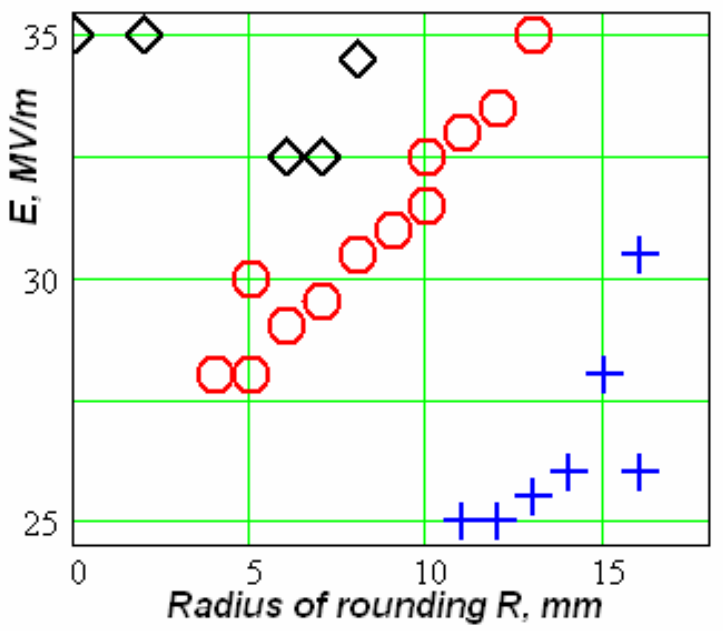

Figure 6: Dependences of $E$ corresponding to max $A$ on $R$.
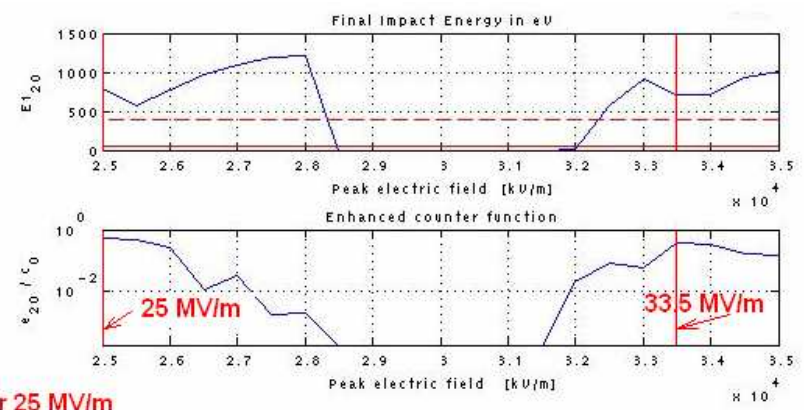

Trajectories for $25 \mathrm{MV} / \mathrm{m}$
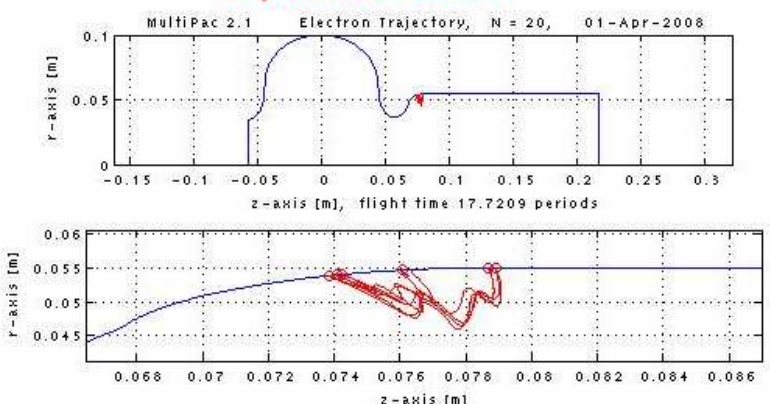

$2-2 \times$ is $[m]$

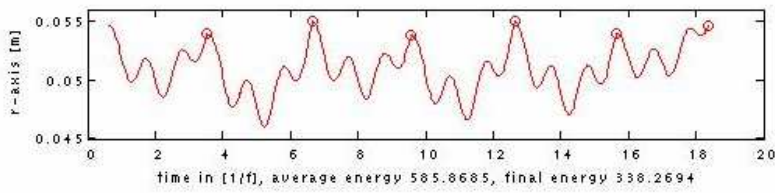

Trajectories for $33.5 \mathrm{MV} / \mathrm{m}$
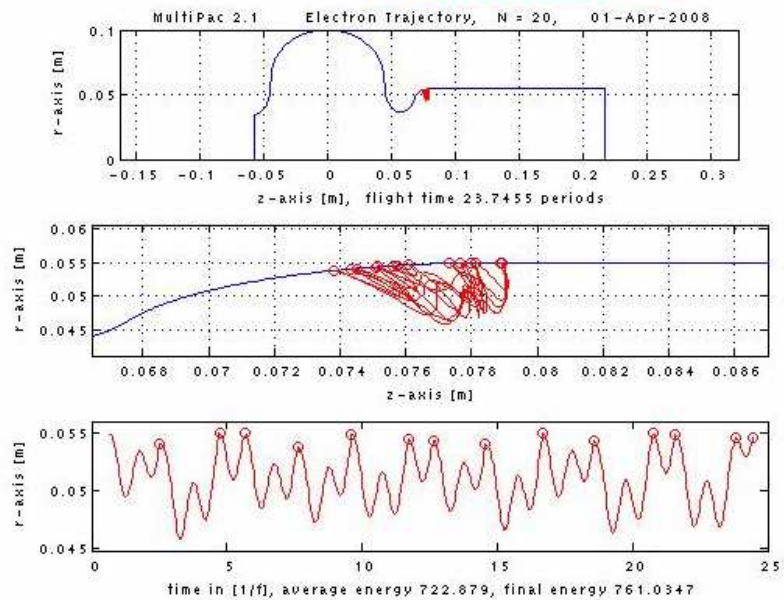

Figure 7: Three- and two-periodic MP at two different amplitudes of the peak electric field for $R=12 \mathrm{~mm}$. 


\section{CAVITY WITH A TAPERED END PORT}

The geometry of the Ichiro cavity is shown in Fig. 8. It has a tapered transition from a larger diameter cavity beam-pipe to a smaller diameter end port. The small distance between the coupler and the flange does not allow to increase the taper length beyond $23 \mathrm{~mm}$. Rounding radii at the ends of the transition cone are not presented in [3], but considering the $45^{\circ}$ angle of the cone slope one can find these radii to be equal to $10.86 \mathrm{~mm}$. While actual dimensions can be slightly different and the presence of the coupler can change somewhat the fields in this transition, the small corrections would not affect the results in a significant way. The calculations show existence of resonant trajectories in the minimum of the electric field, Fig. 9, though, again, the code doesn't show the normalized enhanced counter function of more than 1. Possible MP can occur at the same field providing one takes into account that field in Fig. 9 is the peak surface electric field while in [3] MP zones are plotted as function of the accelerating gradient.

Keeping the length of the taper equal to $23 \mathrm{~mm}$, one can decrease the taper angle by making the rounding radius smaller. But even then the minimum of electric field still exists though it becomes narrower and deeper. The only reasonable way to eliminate the minimum and thus multipacting is to increase the length of the taper transition.

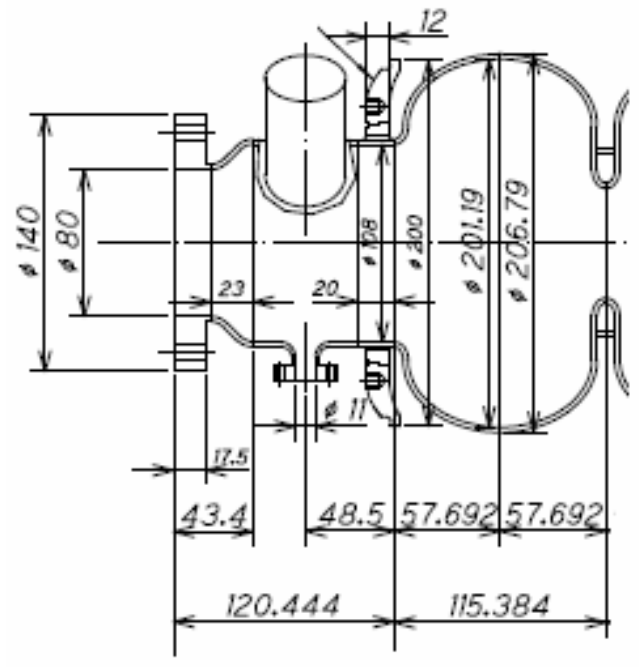

Figure 8: Drawing of the Ichiro cavity end cell with the tapered port [5].

\section{MECHANISM OF THE MOTION}

Motion of electrons near the minimum of RF electric field can be explained by the mechanism suggested in [6]. According to this work, acceleration of electrons in RF field is defined by expressions

$$
\ddot{\mathbf{r}}_{0}(t)=-\nabla \Phi, \quad \Phi=(\eta / 2 \omega)^{2}|\mathbf{E}|^{2},
$$

where $\eta=e / m . \mathbf{r}_{0}(t)$ is a slowly varying (in terms of the $\mathrm{RF}$ oscillation period) function as distinguished from an oscillating function $\mathbf{r}_{1}(t)=\mathbf{A} \sin \omega t$ (we omit terms connected with initial position and velocity), $\Phi$ is the potential proportional to the square of the electric field amplitude. Function $\mathbf{r}_{1}(t)$ is assumed much smaller than the distance over which the amplitude of the RF field changes significantly. As one can see from the above expression, electrons are pushed to the region of lower electric field amplitude (so-called Miller force.) This approach was used in [7] to describe motion of electrons in a coaxial transmission line.

In the case considered in this paper, one has an additional effect. Wherever the electric field amplitude reaches a minimum, there is a potential well. So multipacting electrons are i) pushed toward the minimum of electric field and ii) can be easily trapped in that region if potential well is deep enough. While the condition for smallness of the oscillating function may not always be valid, it does not invalidate the related physical effects, but is only exactness of the formulae.

One can imagine the behavior of electrons in the cavity low electric field region as an electron wind blowing in the direction from the iris to the beam-pipe. In a "calm corner" behind the iris electrons can accumulate and MP can emerge if the secondary emission yield is high enough. Eliminating these "calm corners" can make the cavity free of multipacting.

\section{CONCLUSIONS}

Attraction of multipacting electrons to the minimum of electric field can be explained in terms of the potential well created in the RF field [6]. We would like to point out that multipacting near the cavity equator also exists near the minimum (zero) of the electric field. This is usually one-periodic MP. In the cases analyzed in this paper we see more complex trajectories of MP electrons in the region of minimal $E$.

The fact that the multipacting electrons are attracted to a minimum of electric field, gives us an insight into how to avoid this phenomenon. Namely, the multipactor-free transitions between cavities and beam-pipes should have shapes designed to avoid local minima of the surface electric field. In such transitions electrons, instead of being attracted to "calm corners," will drift in the direction of decaying field.

\section{ACKNOWLEDGEMENT}

The authors are thankful to Hasan Padamsee for helpful discussions and advice.

\section{REFERENCES}

[1] V. Shemelin, "Multipacting in Crossed RF Fields near Cavity Equator," Proc. of EPAC 2004, Lucerne, Switzerland, 2004.

[2] R. L. Geng, et al., "Fabrication and Performance of Superconducting RF Cavities for the Cornell ERL Injector," Proc. of PAC 2007, Albuquerque, NM, 2007

[3] Y. Morozumi. "RF Structure Design and Analysis XXXIII", 18 May 2007, http://lcdev.kek.jp/ILC-AsiaWG/WG5notes/ 
[4] P. Ylä-Oijala, et al., "MultiPac - Multipacting Simulation Package with 2D FEM Field Solver," Proc. of the $10^{\text {th }}$ Workshop on RF Superconductivity, Tsukuba, Japan, 2001.

[5] T. Higo, et al., "Estimation of Transient Lorentz Detuning of ICHIRO Cavity with Helium Jacket," ILCAsia-2007-02, September 17, 2007, http://lcdev.kek.jp/ILCAsiaNotes/
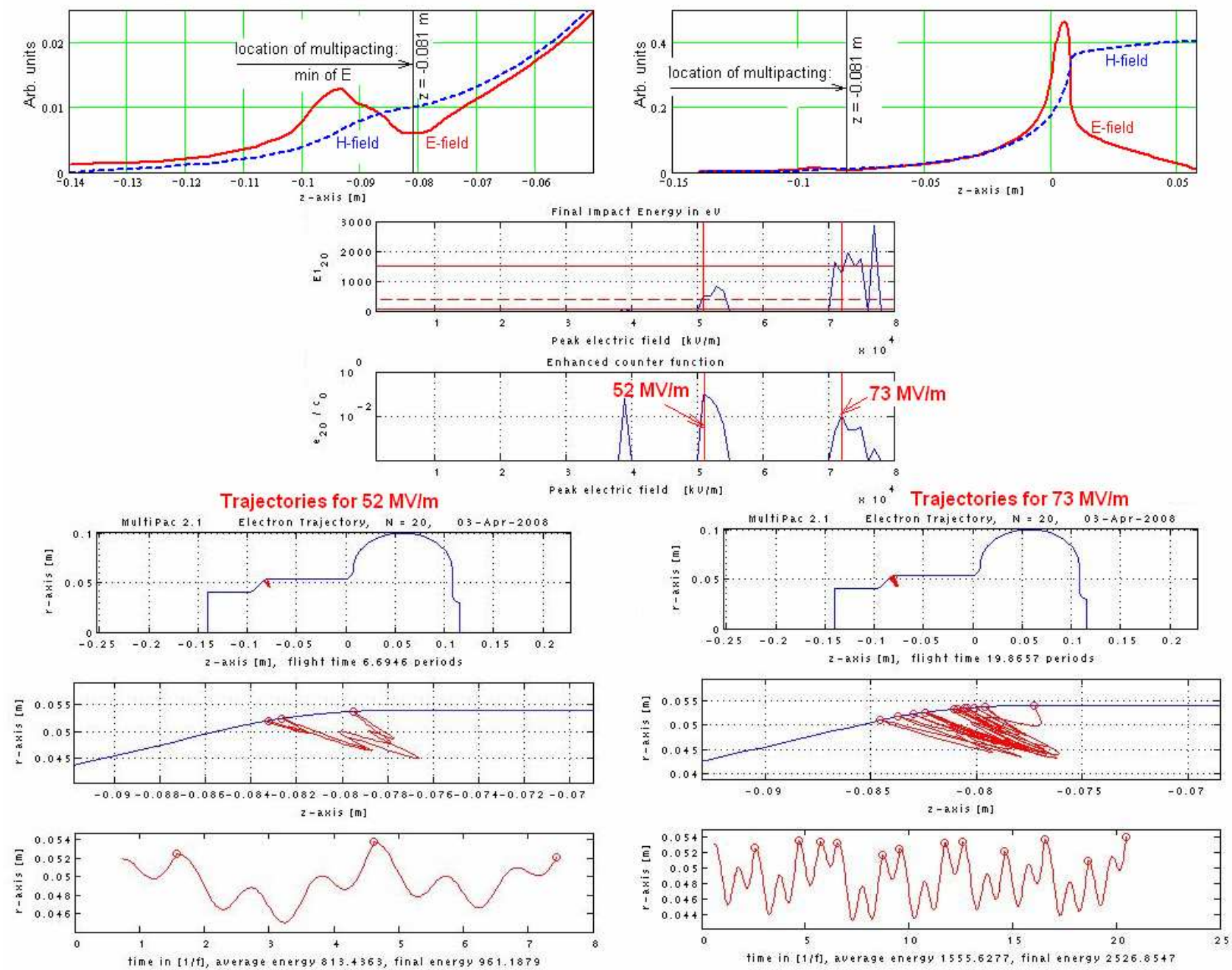

Figure 9: Location of resonant trajectories in the Ichiro cavity. 